\section{Associate Professor of Applied Mechanics, California Institute of Technology, Pasadena, Calif.}

\title{
Random Excitation of a System With Bilinear Hysteresis
}

An analysis is made of the response of a system with bilinear hysteresis to random excitation. It is shown that for moderately large inputs, the additional damping created by the bilinear hysteresis decreases the mean squared deflection compared with that for a linear system with the same viscous damping. However, for large inputs, the decrease in the stiffness of the system due to the bilinear hysteresis causes the mean squared deflection to increase orer that for the equizalent linear system.

\begin{abstract}
$W_{\text {ANy physical systems exhibit bilinear hysteresis of }}$ the type shown in Fig. 1. Hysteresis may be due to the presence of Coulomb friction in the system, or it may be due to elastoplastic behavior of the material in the system. As an example of hysteresis due to Coulomb friction, consider a steel-framed building with masonry walls. The masonry is held in place primarily by its own weight and the resulting frictional forces developed between the masonry blocks. If the building is caused to vibrate, both the masonry and the structural steel work contribute to the stiffness of the building. For small amplitudes of motion, the shearing forces in the masonry are insufficient to overcome the frictional forces, and the masonry contributes considerable shear rigidity to the building. As the amplitude of the motion is increased a point will be reached at which the shearing forces in the masonry exceed the frictional forces, and relative sliding will then occur. During this portion of the motion the shear rigidity of the building is that of the structural steel work; though the masonry no longer contributes to the rigidity of the structure, the relative sliding of the masonry blocks dissipates energy, thereby providing an additional source of damping. Such a system would exhibit bilinear hysteresis of the type shown in Fig. 1. In the past it has been assumed tacitly that such damping would be beneficial and give an additional factor of safety. As the following analysis will show, hysteresis damping of this type may actually lead to larger displacements than would occur in the linear system.
\end{abstract}

\section{Formulation of Problem}

Consider a dynamic system, the restoring force of which exhibits bilinear hysteresis, subjected to a stationary random Gaussian excitation having a white power spectrum; i.e.,

$$
m \ddot{x}+m \beta \dot{x}+k F(x, \mu, t)=N(t)
$$

where

$$
\begin{aligned}
& F(x, \mu, t) \text { is the hysteresic restoring force of Fig. } 1 \\
& F(x, \mu, t) \text { is such that as } \mu \rightarrow 0, F(x, \mu, t) \rightarrow x
\end{aligned}
$$

Let

Contributed by the Applied Mechanics Division for presentation at the Winter-Annual Meeting, New York, N. Y., November 27December 2, 1960, of The American Society of Mechanical Engineers.

Discussion of this paper should be addressed to the Secretary, ASME, 29 West 39th Street, New York, N. Y., and will be accepted until one month after final publication of the paper itself in the Journai of Applied Mechanics. Manuscript received by ASME Applied Mechanics Division; February 15, 1960. Paper No. 60-WA27.

$$
\begin{aligned}
k / m & =\omega_{0}^{2} \\
\omega_{0} t & =\tau \\
N(t) / k & =x_{s}(\tau) \\
\beta / \omega_{0} & =\bar{\beta}
\end{aligned}
$$

Using (2), equation (1) becomes

$$
x^{\prime \prime}+\bar{\beta} x^{\prime}+F(x, \mu, \tau)=x_{s}(\tau)
$$

If in (3) $\mu=0$ and $\bar{\beta}$ is small, the solution may be written

$$
\begin{gathered}
x=R(\tau) \cos [\tau+\theta(\tau)] \\
x^{\prime} \doteq-\omega R(\tau) \sin [\tau+\theta(\tau)]
\end{gathered}
$$

where $R$ and $\theta$ are slowly varying functions of $\tau$. If in (3) $\mu \neq 0$ but is small, the essentially slowly varying nature of the solution will be preserved.

\section{Method of Solution}

The method of solution to be used in this paper is to replace equation (3) by an equivalent linear differential equation, which may then be solved quite readily. Rewrite equation (3) in the following form:

$$
x^{\prime \prime}+\beta_{\mathrm{eq}} x^{\prime}+\omega_{\mathrm{eq}}^{2} x+\epsilon\left(x, x^{\prime}, \tau\right)=x_{s}(\tau)
$$

where $\epsilon\left(x, x^{\prime}, \tau\right)$ is the equation deficiency. If in $(6) \epsilon\left(x, x^{\prime}, \tau\right)$ is neglected, the equation is linear and may be solved readily. The smaller $\epsilon\left(x, x^{\prime}, \tau\right)$, the smaller the error in neglecting it. Hence the logical choice of the system constants $\beta_{\mathrm{eq}}$ and $\omega_{\mathrm{eq}}{ }^{2}$ are those values which minimize the equation deficieney. For mathemati-

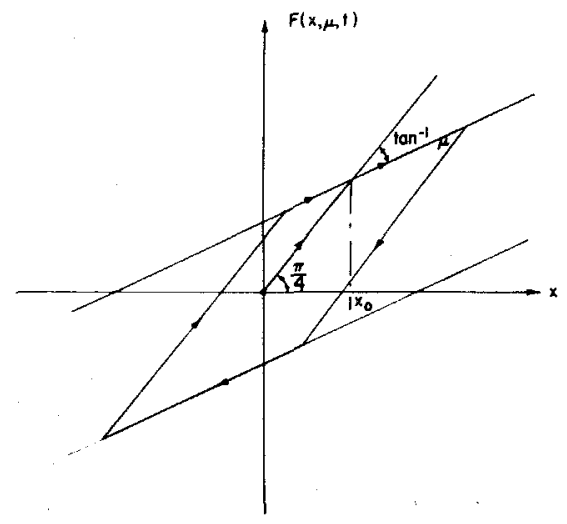

Fig. 1 Bilinear hysteresis 
cal expediency the minimization of the mean squared error will be used. Thus

$$
\overline{\epsilon^{2}\left(x, x^{\prime}, \tau\right)}=\overline{\left[\left(\bar{\beta}-\dot{\beta}_{\mathrm{eq}}\right) x^{\prime}+F(x, \mu, \tau)-\omega_{\mathrm{eq}}{ }^{2} x\right]^{2}}
$$

where the bar superscript denotes average with respect to time.

Minimizing (7) with respect to $\beta_{\mathrm{eq}}$ and $\omega_{\mathrm{eq}}{ }^{2}$, gives

$$
\begin{gathered}
\beta_{\mathrm{eq}}=\overline{\left[\bar{\beta}\left(x^{\prime}\right)^{2}+x^{\prime} F(x, \mu, \tau)-\omega_{\mathrm{eq}}{ }^{2} x x^{\prime}\right]} \overline{\left[\left(x^{\prime}\right)^{2}\right]^{-1}} \\
\omega_{\mathrm{eq}}{ }^{2}=\overline{\left[\left(\bar{\beta}-\beta_{\mathrm{eq}}\right) x^{\prime} x+x F^{\prime}(x, \mu, \tau)\right]} \overline{\left[(x)^{2}\right]}{ }^{-1}
\end{gathered}
$$

Now, for a stationary, differentiable random process, the velocity and displacement are uncorrelated at the same time; thus

$$
\overline{x x^{\prime}}=0
$$

Hence equations (8) and (9) become

$$
\begin{gathered}
\beta_{\mathrm{eq}}=\bar{\beta}+\overline{\left[x^{\prime} F(x, \mu, \tau)\right]} \overline{\left[\left(x^{\prime}\right)^{2}\right]}-1 \\
\omega_{\mathrm{eq}}{ }^{2}=\overline{[x F(x, \mu, \tau)]}\left[\overline{\left[(x)^{2}\right.}\right]^{-1}
\end{gathered}
$$

If in equation (6) $\epsilon\left(x, x^{\prime}, \tau\right)$ is neglected, the equation is linear; further, if $\bar{\beta}$ and $\mu$ are small, $\beta_{\mathrm{eq}}$ will also be small, hence the solution may be written:

$$
\begin{aligned}
x & =R(\tau) \cos \left[\omega_{\mathrm{eq}} \tau+\theta(\tau)\right] \\
x^{\prime} & =-\omega_{\mathrm{eq}} R(\tau) \sin \left[\omega_{\mathrm{eq}} \tau+\theta(\tau)\right]
\end{aligned}
$$

where $R$ and $\theta$ are slowly varying functions of $\tau$. Since $R$ and $\theta$ are slowly varying quantities, the integrands in (11) and (12) may be replaced by their average values over one cycle. Hence

$$
\begin{gathered}
\beta_{\mathrm{eq}}=\bar{\beta}+\left[\overline{\left[\frac{1}{2 \pi} \int_{0}^{2 \pi}-\omega_{\mathrm{eq}} R \sin \psi F(R \cos \psi, \mu, \tau) d \psi\right]}\right. \\
\omega_{\mathrm{eq}}^{2}=\left[\frac{\left.1 / 2 \omega_{\mathrm{eq}}{ }^{2} \overline{R^{2}}\right]^{-1}}{\left[\frac{1}{2 \pi} \int_{0}^{2 \pi} R \cos \psi F(R \cos \psi, \mu, \tau) d \psi\right]\left[1 /{ }_{2} \overline{R^{2}}\right]^{-1}}\right.
\end{gathered}
$$

where

$$
\psi=\left[\omega_{\mathrm{eq}} \tau+\theta\right]
$$

Define:

$$
\begin{aligned}
& C(R)=\frac{1}{\pi} \int_{0}^{2 \pi} \cos \psi F(R \cos \psi, \mu, \tau) d \psi \\
& S(R)=\frac{1}{\pi} \int_{0}^{2 \pi} \sin \psi F(R \cos \psi, \mu, \tau) d \psi
\end{aligned}
$$

These integrals have been evaluated previously by the author ${ }^{1}$

Using (17) and (18) equations (14) and (15) become

$$
\begin{gathered}
\left.\beta_{\mathrm{eq}}=\bar{\beta}-\overline{[R S(R)}\right]\left[\omega_{\mathrm{eq}} \overrightarrow{R^{2}}\right]^{-1} \\
\omega_{\mathrm{eq}}{ }^{2}=[\overrightarrow{R C(R)}]\left[\overrightarrow{R^{2}}\right]^{-1}
\end{gathered}
$$

If the process is an ergodic one, the time averages may be replaced by the stochastic averages, hence equations (19) and (20) may be written

$$
\begin{gathered}
\beta_{\mathrm{eq}}=\bar{\beta}-\left[\int_{0}^{\infty} R S(R) P(R) d R\right]\left[\omega_{\mathrm{eq}} \int_{0}^{\infty} R^{2} P(R) d R\right]^{-1} \\
\omega_{\mathrm{eq}}{ }^{2}=\left[\int_{0}^{\infty} R C(R) P(R) d R\right]\left[\int_{0}^{\infty} R^{2} P(R) d R\right]^{-1}
\end{gathered}
$$

${ }^{1}$ T. K. Caughey, "Sinusoidal Excitation of a System With Bilinear Hysteresis," to be published in the Journal. of Applied Mechanics. ASME Paper No. 60-APM-8. where $P(R) d R$ is the probability that $R$ lies in the range $R$ to $R$ $+d R$.

If in equation (6) $\mu$ is set equal to zero, the equation is linear, and since $x_{s}(\tau)$ is Gaussian $x(\tau)$ and $x^{\prime}(\tau)$ will also be Gaussian. Further, if the damping is small, such that equations (4) and (5) hold, then the probability density function $(P) R$ will be the Rayleigh distribution. In the nonlinear system the statistics of the solution will not, in general; be Gaussian; however, if the nonlinearity is small the statistics will be approximately Gaussian. Hence

$$
P(R) d R \simeq \frac{R}{\sigma^{2}} e^{-R^{2 / 2 \sigma^{2}} d R}
$$

where

$$
\sigma^{2}=\widetilde{x^{2}}=\left\langle x^{2}\right\rangle_{\mathrm{avg}}
$$

Equations (21) and (22) now become

$$
\begin{aligned}
\beta_{\mathrm{eq}}=\bar{\beta}-\left[\int_{0}^{\infty} \frac{R^{2}}{\sigma^{2}} S(R) e^{-R^{2} / 2 \sigma^{2}} d R\right] \\
\omega_{\kappa \mathrm{q}^{2}}=\left[\int_{0}^{\infty} \frac{R^{2}}{\sigma^{2}} C(R) e^{-R^{2} / 2 \sigma^{2}} d R\right] \\
{\left[\int_{0}^{\infty} \frac{R^{3}}{\sigma^{2}} e^{-R^{2} / 2 \sigma^{2}} d R\right]^{-1} } \\
{\left[\sigma^{2} e^{-R^{2} / 2 \sigma^{2}} d R\right]^{-1} }
\end{aligned}
$$

\section{Evaluation of $S(R)$ and $C(R)$}

Iset

$$
\cos \theta^{*}=\left(1-\frac{x_{0}}{2 R}\right)
$$

Using Fig. 1, $S(R)$ and $C(R)$ may be evaluated readily

$$
\begin{aligned}
S(R)=\frac{2}{\pi} & {\left[\int_{0}^{\theta^{*}}\left[\mu x_{0}-\mu R+R \cos \psi\right] \sin \psi d \psi\right.} \\
& \left.+\int_{\theta^{*}}^{\pi}\left[-\mu x_{0}+R(1-\mu) \cos \psi\right] \sin \psi d \psi\right]
\end{aligned}
$$

Therefore

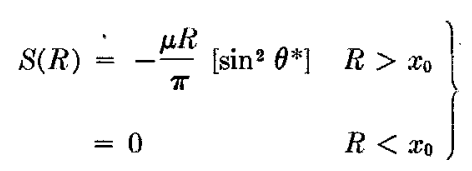

$$
\begin{aligned}
C(R)=\frac{2}{\pi} & {\left[\int_{0}^{\theta^{*}}\left[\mu x_{0}-\mu R+R \cos \psi\right] \cos \psi d \psi\right.} \\
& \left.\quad+\int_{\theta^{*}}^{\pi}\left[-\mu x_{0}+R(1-\mu) \cos \psi\right] \cos \psi d \psi\right]
\end{aligned}
$$

Therefore

$$
\left.\begin{array}{rr}
C(R)=\frac{R}{\pi}\left[\mu \theta^{*}+(1-\mu) \pi-\frac{\mu}{2} \sin 2 \theta^{*}\right] \\
R>x_{0} \\
=R & R<x_{0}
\end{array}\right\}
$$

Substituting (28) into (24)

$$
\beta_{\mathrm{eq}}=\bar{\beta}+\frac{1}{2 \sigma^{4} \omega_{\mathrm{eq}}} \int_{x_{0}}^{\infty} \frac{\dot{\mu} R^{3}}{\pi} \sin ^{2} \theta^{*} e^{-R^{2} / 2 \sigma^{2}} d R
$$


Using (26), (31) may be integrated to give

$$
\beta_{\mathrm{eq}}=\bar{\beta}+\frac{2}{\sqrt{\pi}} \frac{\mu}{\omega_{\mathrm{eq}}} \frac{x_{0}}{\sqrt{2 \sigma}} \operatorname{erfe} \frac{x_{0}}{\sqrt{2 \sigma}}
$$

where erfe is the complimentary error function. Let

$$
\begin{aligned}
& n=\frac{\sqrt{2} \sigma_{0}}{x_{0}} \\
& y=\frac{\sigma}{\sigma_{0}} \\
& \lambda=n^{2} y^{2}
\end{aligned}
$$

where $\sigma_{i j}=\sqrt{x^{2}}$ when $\mu=0$. Using (33), (32) becomes

$$
\beta_{\mathrm{eq}}=\bar{\beta}+\frac{2 \mu}{\sqrt{ } \pi} \frac{1}{\omega_{\mathrm{eq}} \lambda^{1 / 2}} \operatorname{erfc}\left(\frac{1}{\lambda^{1 / 2}}\right)
$$

Equation (34) may be expressed in a more convenient form for plotting as

$$
\left(\beta_{\text {eq }}-\bar{\beta}\right) \omega_{\text {eq }} / \mu=2(\pi \lambda)^{-1 / 2} \operatorname{erfc}\left(\lambda^{-1 / 2}\right)
$$

The function defined by equation (35) is shown in Fig. 2. It will be observed that the function is zero when $\lambda$ is zero, reaches its maximum at about $\lambda$ equal to 3 , then tends asymptotically to zero.

Substituting (30) into equation (25):

$$
\begin{aligned}
\omega_{\mathrm{eq}}^{2} & =\frac{1}{2 \pi \sigma^{4}}\left[\int_{0}^{x_{0}} R^{3} \pi e^{-R^{2} / 2 \sigma^{2}} d R\right. \\
& \left.+\int_{x^{\infty}}^{\infty} R^{3}\left[\pi-\mu\left(\pi-\theta^{*}+1 / 2 \sin 2 \theta^{*}\right)\right] e^{-R^{2} / 2 \sigma^{2}} d R\right]
\end{aligned}
$$

Let

$$
R / x_{0}=z
$$

Substituting (37) into (36) and integrating the second term by parts, gives

$\omega_{\mathrm{eq}}^{2}=1-\frac{8 \mu}{\pi} \int_{1}^{\infty}\left[(z \lambda)^{-1}(z-1)^{1 / 2}\right.$

$$
\left.+z^{-3}(z-1)^{1 / 2}\right] e^{-z^{2} / \lambda} d z
$$

Equation (38) may be written in a more convenient form for plotting as

$$
\begin{aligned}
\frac{1-\omega_{\mathrm{eq}}^{2}}{\mu}=\frac{8}{\pi} \int_{1}^{\infty}\left[(z \lambda)^{-1}(z-\right. & 1)^{1 / 2} \\
& \left.+z^{-3}(z-1)^{1 / 2}\right] e^{-z^{2} / \lambda} d z
\end{aligned}
$$

The integral (39) has been evaluated numerically and the results

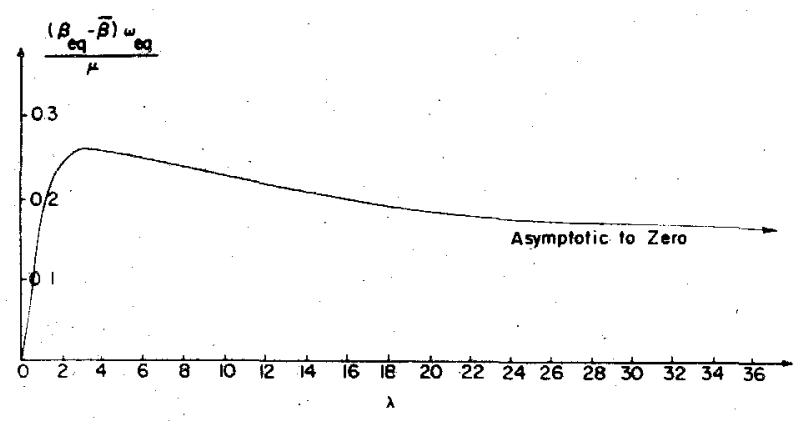

Fig. 2 are plotted in Fig. 3. It will be observed that the function starts at zero for $\lambda$ equal to zero, increases with $\lambda$, and tends asymptotically to 1 as $\lambda$ tends to infinity.

\section{Mean Squared Displacement}

If in equation (6), the deficiency term $\dot{\epsilon}\left(x, x^{\prime}, \tau\right)$ is neglected, the equation is linear and may be solved readily.

If $X(\omega)$ is the power spectrum of $x(\tau), X_{s}(\omega)$ is the power spectrum of $x_{s}(\tau)$. Then

$$
\begin{aligned}
X(\omega) & =\frac{X_{s}(\omega)}{\left[\omega_{\mathrm{eq}}^{2}-\omega^{2}\right]^{2}+\left[\omega \beta_{\mathrm{eq}}\right]^{2}} \\
\sigma^{2} & =\overline{x^{2}}=\int_{0}^{\infty} X(\omega) d \omega
\end{aligned}
$$

If $x_{s}(\tau)$ has a white spectrum of density $4 D /$ cycle, then

$$
\sigma^{2}=\frac{D}{\omega_{\mathrm{eq}}^{2} \beta_{\mathrm{eq}}}
$$

Let

$$
D / \bar{\beta}=\sigma_{0}^{2}=\overline{x^{2}}
$$

when $\mu=0$. Hence

$$
\frac{\sigma^{2}}{\sigma_{0}^{2}}=y^{2}=\frac{\bar{\beta}}{\omega_{\mathrm{eq}}^{2} \beta_{\mathrm{eq}}}
$$

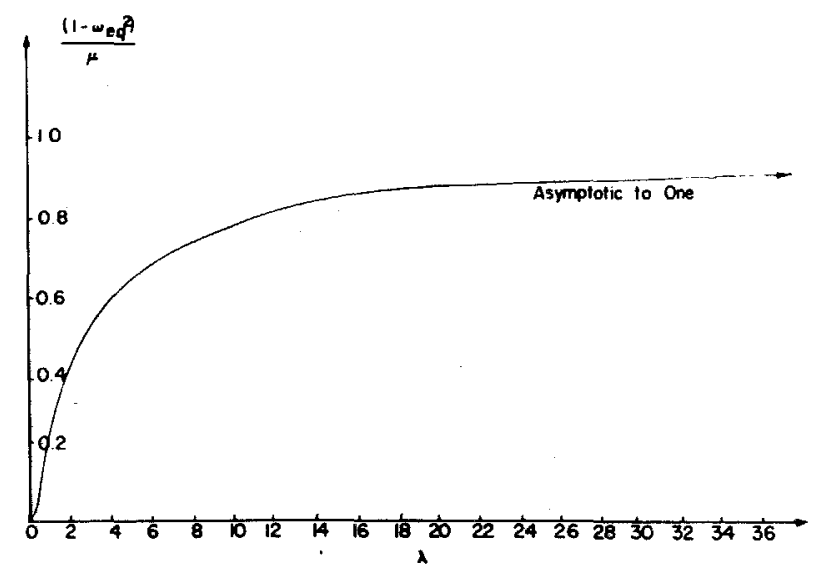

Fig. 3

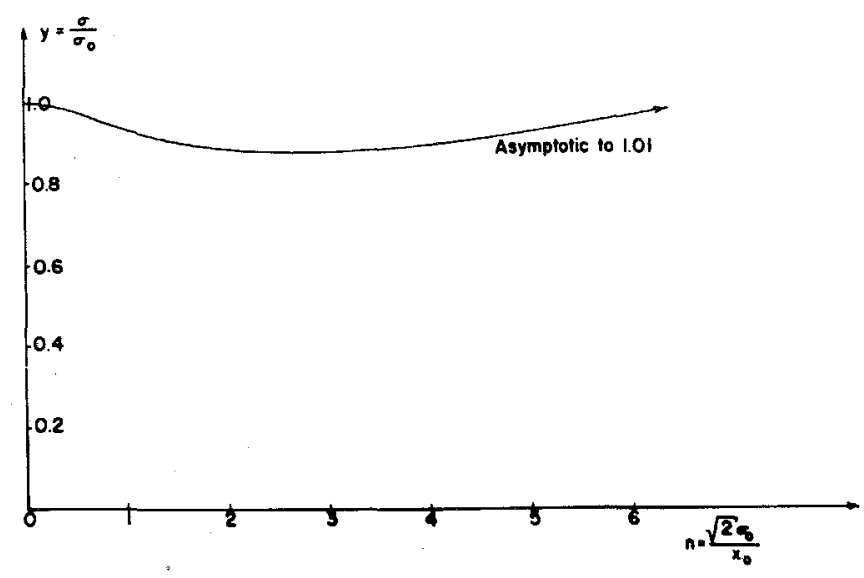

Fig. 4 


\section{Method of Solving Equation (43)}

In (43), $\omega_{\mathrm{eq}}{ }^{2}$ and $\beta_{\mathrm{eq}}$ are functions of $\lambda=n^{2} y^{2}$; therefore, assume a value of $\lambda$, obtain $\omega_{\mathrm{eq}}{ }^{2}$ and $\beta_{\mathrm{eq}}$ from Figs. 2 and 3 , solve (43) for $y^{2}$, then $n^{2}=\lambda / y^{2}$. Repeat for different values of $\lambda$, plot $y$ against $n$.

Results. Fig. 4 shows the results for the case where $\mu=\bar{\beta}=$ 0.02 . It will be observed that bilinear hysteresis has the following effects:

1 . For small values of $n$, there is no effect.

2 For moderate values of $n$, there is an appreciable reduction in the rms deflection.

3 For large values of $n, y$ tends asymptotically to $1 /(1-\mu)^{1 / 2}$.

\section{Conclusions}

From the foregoing analysis it will be seen that the effects of bilinear hysteresis are beneficial for small or moderately large random inputs. For large inputs, however, the effect of bilinear hysteresis is to increase root mean square deflection compared with that for the linear system $\mu=0$. The explanation of this apparent paradox is that for large inputs, the damping tends asymptotically to the linear damping $\bar{\beta}$ while the effective stiffness of the system tends asymptotically to $(1-\mu)$. The combination of damping $\vec{\beta}$ and stiffness $(1-\mu)$ results in a mean squared deflection which is $1 /(1-\mu)$ times the mean squared deflection for the linear system $(\mu=0)$. In the case of an elastoplastic structure, $\mu=1$, the results would be disastrous. It must therefore be concluded that too much reliance should not be placed in the usefulness of bilinear hysteresis as a means of increasing the effective damping in structures subjected to random excitation such as earthquakes. 